\title{
MYCOBATERIUM BOVIS ARACHNOIDITIS
}

Temple ${ }^{\prime}$ Street CHILDREN'S UNIVERSTTY HOSPITAL
C. Ó Maoldomhnaigh¹, R. Kernan¹, L. Baker², D. Crimmins³, E. Moylett ${ }^{4}$, K. Butler ${ }^{1}$, P. Gavin ${ }^{1}$.

${ }^{1}$ Children's University Hospital- Temple Street, Paediatric Infectious Diseases, ${ }^{2}$ Paediatrics, and ${ }^{3}$ Neurosurgery Departments, Dublin, and ${ }^{4}$ Galway University Hospital, Department of Paediatrics, Galway, Ireland.

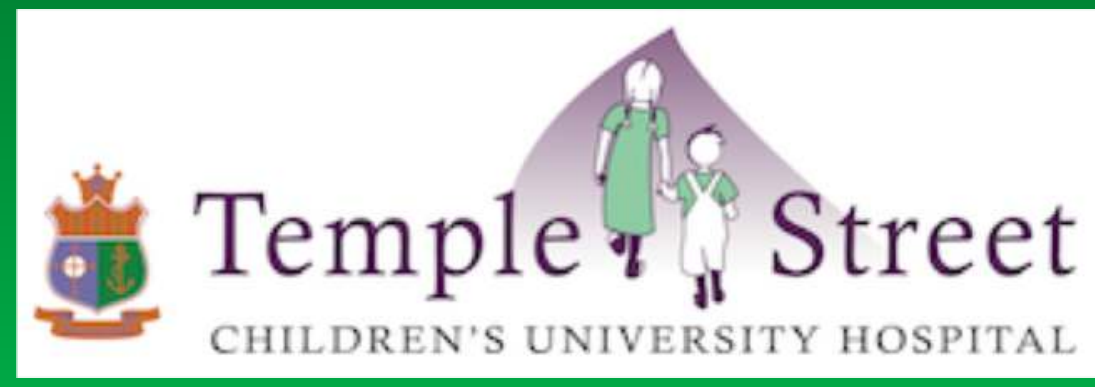

\section{Background}

- Tuberculous meningitis is rare in areas of low TB incidence.

- Diagnosis is challenging due to non-specific presenting symptoms, difficulty in culturing the organism and low yield of CSF PCR.

\section{Case presentation}

- An 11-year old, HIV-negative, caucasian Irish girl presented with 2-weeks of intermittent fever, worsening headaches and vomiting.

- She was previously well, without history of travel, known TB contacts, exposure to farm animals or unpasteurised dairy products.

- Admission bloods and CT brain were normal. LP parameters are shown in the table.

- CSF Gram and AFB stains, culture and multiplex bacterial PCR were negative.

- Empiric ceftriaxone and aciclovir were started. An EVD was inserted for worsening encephalopathy and increased ventricular dilatation on repeat CT brain.

- CSF AFB stain, Filmarray, and MTB complex PCR were again negative.

\begin{tabular}{|c|c|c|c|c|}
\hline & $\begin{array}{c}\text { WCC } / \mathrm{cmm} \\
\text { ( lymphocyte } \%)\end{array}$ & RCC /cmm & Protein mg/L & Glucose mmol/L \\
\hline Lumbar puncture D3 & $443(77 \%)$ & 143 & $>2500$ & 1.7 \\
\hline CSF from EVD D5 & $62(89 \%)$ & 31 & 330 & 3.1 \\
\hline
\end{tabular}

- The discrepancy in lumbar and ventricular CSF parameters prompted MRI brain and spine (Figure) with contrast.

- This showed extensive enhancement of spinal cord subarachnoid space and brainstem and basal cistern pial surfaces.

- She underwent lumbosacral laminectomy and nerve root biopsy for suspected malignancy.

- Histology was negative for malignancy but showed granulomas and a single AFB stain positive organism.

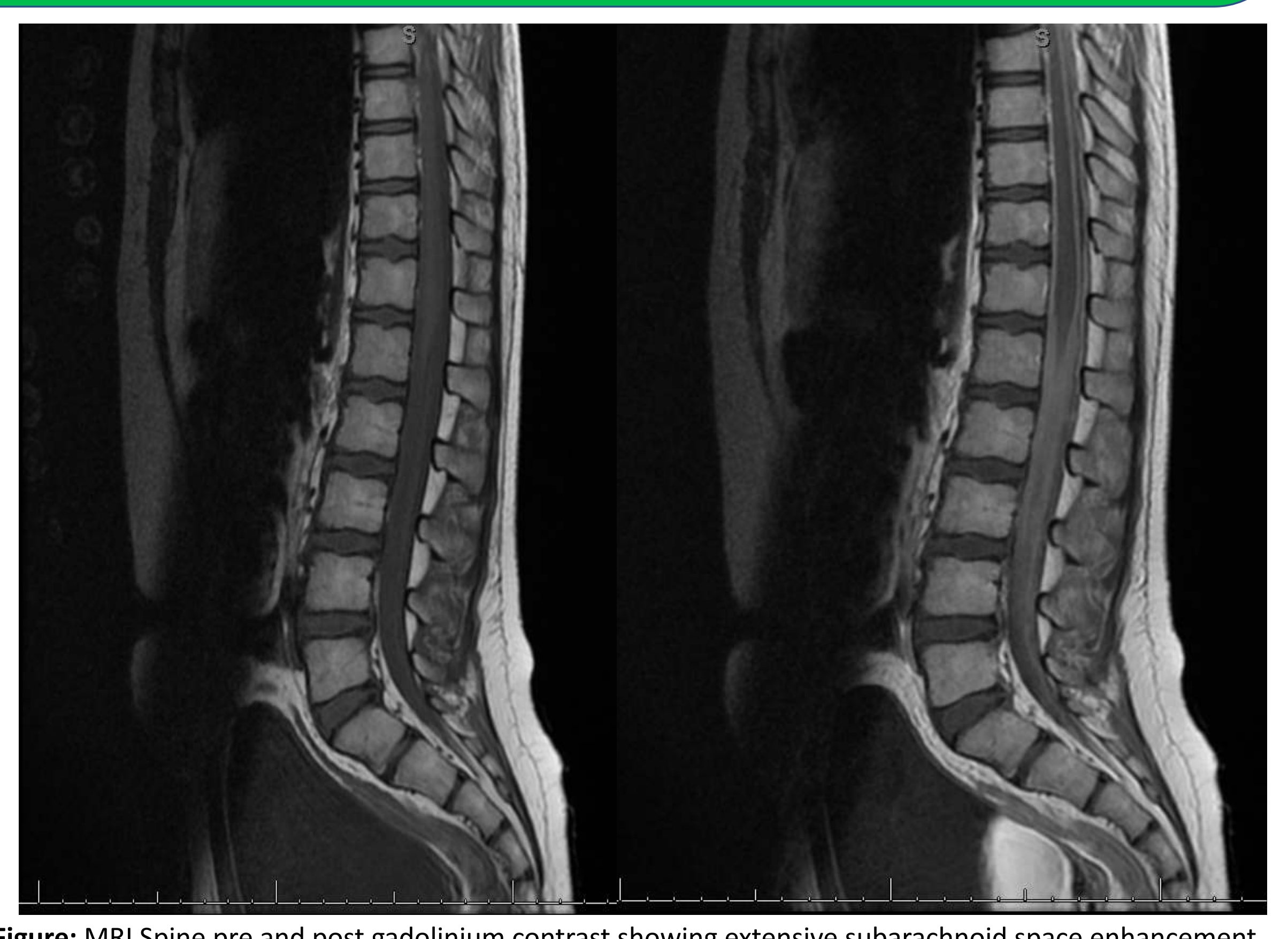

Figure: MRI Spine pre and post gadolinium contrast showing extensive subarachnoid space enhancement.

- Anti-mycobacterial chemotherapy and high dose dexamethasone were commenced for presumed MTB infection, confirmed on positive MTB complex PCR and subsequent culture identification as Mycobaterium bovis.

- She is now well 6 months into treatment.

Learning Points/Discussion

- This case underlines the difficulties inherent in diagnosis of tuberculous meningitis and particularly arachnoiditis.

- Repeat CSF MTB complex PCR samples were negative with diagnosis ultimately depending on spinal neuroimaging and tissue biopsy. 